\title{
BENIGN, PATHOGENIC AND COPY NUMBER VARIATIONS OF UNKNOWN CLINICAL SIGNIFICANCE IN PATIENTS WITH CONGENITAL MALFORMATIONS AND DEVELOPMENTAL DELAY
}

\author{
Mihaylova $\mathrm{M}^{1, *}$, Staneva $\mathrm{R}^{1,2}$, Toncheva $\mathrm{D}^{1}$, Pancheva $\mathrm{M}^{1,2}$, Hadjidekova $\mathrm{S}^{1,2}$
}

*Corresponding Author: Dr. Marta Mihaylova, Department of Medical Genetics, Medical Faculty, Medical University of Sofia, 2 Zdrave Street, 1431 Sofia, Bulgaria. Tel: +3592-9172-735. E-mail: marta.mih@gmail.com

\begin{abstract}
The high frequency (3.0-5.0\%) of congenital anomalies (CA) and intellectual disabilities (IDs), make them a serious problem, responsible for a high percentage (33.0\%) of neonatal mortality. The genetic cause remains unclear in $40.0 \%$ of cases. Recently, molecular karyotyping has become the most powerful method for detection of pathogenic imbalances in patients with multiple CAs and IDs. This method is with high resolution and gives us the opportunity to investigate and identify candidate genes that could explain the genotype-phenotype correlations. This article describes the results from analysis of 81 patients with congenital malformations (CMs), developmental delay (DD) and ID, in which we utilized the CytoChip ISCA oligo microarray, $4 \times 44 \mathrm{k}$, covering the whole genome with a resolution of $70 \mathrm{~kb}$. In the selected group of patients with CAs, 280 copy number variations (CNVs) have been proven, 41 were pathogenic, 118 benign and 121 of unknown clinical significance (average number of variations 3.5). In six patients with established pathogenic variations, our data revealed eight pathogenic aberrations associated with the corresponding phenotype. The interpretation of the other CNVs was made on the basis of their frequency in the investigated group, the size of the variation, content of genes in the region and the type of the CNVs (deletion or duplication).
\end{abstract}

Keywords: Microarray comparative genomic hybridization (aCGH); Congenital anomalies (CAs); Copy number variations (CNVs).

\footnotetext{
${ }^{1}$ Department of Medical Genetics, Medical Faculty, Medical University of Sofia, Sofia, Bulgaria

2 Woman Health Hospital "Nadezhda", Sofia, Bulgaria
}

\section{INTRODUCTION}

A high percentage of genetic diseases manifest themselves in the first 28 days after birth, but the full clinical symptoms may not be evident in newborns. Conventional diagnostic tests are with low resolution. They are also time-consuming, labor-intensive and are difficult to automate, therefore, the etiology of congenital anomalies (CAs) in $40.0-60.0 \%$ of the cases remains unclear. Molecular and molecular cytogenetic techniques such as FISH (fluorescent in situ hybridization), QF-PCR (quantitative fluorescence-polymerase chain reaction), MLPA (multiplex ligation-dependent probe amplification), have enhanced the diagnosis, but they require preliminary information on the suspected chromosomal abnormality and on the location. The new genomic technologies such as micro- array comparative genomic hybridization $(\mathrm{aCGH})$ and nextgeneration sequencing (NGS), combine the advantages of conventional technologies, enabling whole-genome screening in high resolution.

At present, great interest has arisen in a newly discovered variation known as copy number variations (CNVs), which, in 2007, was indicated to be the "breakthrough" of the year [1]. Currently, CNVs are defined as a ubiquitous segment of DNA longer than $1 \mathrm{~kb}$, presented at a variable copy number, compared to the reference genome. The great interest in CNVs was aroused by the observation that they can affect transcriptional activity and translational levels of adjacent genes [2-8]. An association was found between certain CNVs and predisposition to multifactorial diseases [3,9-15]. Copy number variations differ in type and frequency in different populations and can be used as a valuable source of information for the study of genetic characteristics of the nations [16-20]. With the accumulation of huge amounts of data for different CNVs, certain phenotype-genotype correlations have been defined [21]. 
It is therefore possible for CNVs that have been initially considered benign to later be proved as increasing sensitivity to multifactorial disease or causing genetic disease with late onset or incomplete penetrance. Therefore, the term CNVs is generally used to describe the CNVs both in patients and in healthy controls, as well as to describe the genomic imbalances, causing known microdeletion/ micro-duplication syndromes. Consequently, CNVs can be divided into benign, pathogenic and CNVs of unknown clinical significance [22-25] and can be polymorphic with frequency greater than $1.0 \%$ or rare, less than $1.0 \%$. They can also arise as de novo anomalies or be inherited, multiallelic or biallelic [26]. According to the data from two large studies in healthy populations, the human genome contains $12 \mathrm{CNVs}$ on average [27,28]. Another study revealed that $12.0 \%$ of the human genome is represented by CNVs covering hundreds of genes, disease loci, functional elements and segmental duplications [29]. Sequencing of the human genome revealed that insertions and deletions in absolute terms are responsible for $22.0 \%$ of the variations and cover $74.0 \%$ of the affected nucleotides [30]. Recent data indicate that the genomes of two individuals may vary between 1.0 and $3.0 \%$ [31].

Caramaschi et al. [32] carried out a survey of 116 patients. The detected CNVs were analyzed for genotype/ phenotype correlations with the clinical features of the patients. Pathogenic CNVs (21 deletions, three duplications and three cases with both of them) were observed in 27 patients $(23.3 \%)$. The analysis showed a significant association between pathogenic CNVs and the first appearance of the symptoms before the age of 1 year and the presence of malformations [32]. According to another study on CNVs in 2500 individuals, it was found that in 65.0 to $80.0 \%$ of the cases, CNVs were greater than $100 \mathrm{~kb}$, in 5.0-10.0\% greater than $500 \mathrm{~kb}$, and in $1.0-2.0 \%$ greater than $1 \mathrm{Mb}$ [19]. In addition, the biggest part of the genomic variations were found with a frequency of 0.02 to $1.0 \%$, comprising $6.0 \%$ of our genome. At the same time, polymorphic CNVs represented $0.09 \%$. Another study conducted by Pinto et al. [20] on a healthy population detected that $160 \mathrm{Mb}(\sim 5.0 \%)$ was represented by CNVs, from which $96.0 \%$ were rare, less than $2.0 \%$, while others were common. In this study, we present our findings with regard to the distribution, frequency and clinical significance of the detected CNVs.

\section{MATERIALS AND METHODS}

The present study focuses on 81 patients of both sexes with dysmorphic features, with or without intellectual disability, behavioral problems, failure to thrive, neurological disorders. All of them presented a normal karyotype. The blood was taken in compliance with the standard procedures for good laboratory practice. The genomic screening, arraybased comparative genomic hybridization (aCGH) was performed by standard protocol using the CytoChip ISCA oligo microarray, $4 \times 44$ k (BlueGnome Ltd., Cambridge, Cambridgeshire, UK), resolution $70 \mathrm{~kb}$. Data were analyzed with the BluefuseMulti v.4.2 software (BlueGnome Ltd.).

\section{RESULTS}

In the investigated group, pathogenic CNVs were found in 31 patients. In addition, in the majority of all surveyed individuals, we identified benign CNVs (58/81 patients) and variations of unknown clinical significance (66/81 patients). There were $280 \mathrm{CNVs}$ detected, of which 41 were pathogenic (28 deletions, 13 duplications), 118 benign (91 deletions, 27 duplications) and 121 of unknown clinical significance (50 deletions, 71 duplications). Copy number variations have not been established in six of the patients. The size of all identified CNVs was from 100,021 to $13,881,527 \mathrm{bp}$. There were 169 deletions and $111 \mathrm{du}-$ plications. The largest duplication covered 13,881,527 $\mathrm{bp}$, and the smallest included 100,021 bp. The region of the smallest deletion covered 102,202 bp, and the size of the largest in four $(\mathrm{q} 34.3 \mathrm{q} 35.2)$ region: $\operatorname{arr} 4 \mathrm{q} 34.3 \mathrm{q} 35.2$ $(178,213,959-190,896,645) \times 1$, was $12,682,687$ bp. We revealed eight pathogenic variations associated with the phenotype in six patients from the total group. Variations were distributed over eight chromosomes (chromosomes $2,4,5,10,12,15,17,22)$. In two of the analyzed patients, a combination of two pathogenic CNVs on different chromosomes was found, and four of the patients had a single pathogenic variation. Six of the aberrations were deletions and two of them were duplications. The amount of detected genomic pathogenic changes ranges from $300 \mathrm{~kb}$ to $13 \mathrm{Mb}$. In five patients of the total group, we discovered five pathogenic CNVs that were not associated with the observed phenotype (Table 1).

As well as pathogenic variations, benign CNVs and CNVs of unknown clinical significance were found. There were 239 (an average of 2.9 per patient variations of the total group). These CNVs were distributed over 75 patients. All established benign and unknown CNVs ranged in size from 106,847 to $1,348,283 \mathrm{bp}$. From the total number of identified CNVs (239 without pathogenic CNVs), 179 (74.9\%) covered $100-500 \mathrm{~Kb}, 52(21.8 \%)$, from $500 \mathrm{~Kb}$ to $1 \mathrm{Mb}$ and eight $(3.3 \%)$ were larger than $1 \mathrm{Mb}$. There were 141 deletions and 98 duplications. One hundred and eighteen of the CNVs were benign. They were divided by 
Table 1. Summarized results with pathogenic findings identified after microarray comparative genomic hybridization and associated with the phenotype of the patient.

\begin{tabular}{|c|c|c|c|c|c|c|c|}
\hline \multirow{2}{*}{ Patient } & \multirow{2}{*}{ Chromosome } & $\begin{array}{c}\text { Type of } \\
\text { Aberration }\end{array}$ & \multicolumn{2}{|c|}{ Position (bp) } & \multicolumn{2}{c|}{ Size (bp) } & \multicolumn{2}{c|}{ Cytoband } \\
\hline & & & Start & End & & Start & End \\
\hline \multirow{2}{*}{79} & 2 & deletion & $50,982,143$ & $51,314,401$ & 332,259 & $2 \mathrm{p} 16.3$ & $2 \mathrm{p} 16.3$ \\
& 5 & deletion & $175,470,501$ & $177,136,261$ & $1,665,761$ & $5 \mathrm{q} 35.2$ & $5 \mathrm{q} 35.3$ \\
\hline 52 & 10 & deletion & $122,804,780$ & $135,434,149$ & $12,629,370$ & $10 \mathrm{q} 26.12$ & $10 \mathrm{q} 26.3$ \\
\hline \multirow{2}{*}{46} & 4 & deletion & $178,213,959$ & $190,896,645$ & $12,682,687$ & $4 \mathrm{q} 34.3$ & $4 \mathrm{q} 35.2$ \\
& 12 & duplication & 230,451 & $14,111,977$ & $13,881,527$ & $12 \mathrm{p} 13.33$ & $12 \mathrm{p} 13.1$ \\
\hline 41 & 17 & deletion & $34,450,435$ & $36,248,889$ & $1,798,455$ & $17 \mathrm{q} 12$ & $17 \mathrm{q} 12$ \\
\hline 35 & 22 & deletion & $21,561,492$ & $22,905,039$ & $1,343,548$ & $22 \mathrm{q} 11.21$ & $22 \mathrm{q} 11.22$ \\
\hline 30 & 15 & duplication & $22,765,658$ & $29,030,488$ & $6,264,831$ & $15 \mathrm{q} 11.2$ & $15 \mathrm{q} 13.1$ \\
\hline
\end{tabular}

Table 2. Number and size of detected benign copy number variations and copy number variations of unknown clinical significance.

\begin{tabular}{|l|c|c|c|c|}
\hline Parameters & Total Number & $\mathbf{1 0 0 - 5 0 0 ~ k b}$ & $\mathbf{5 0 0} \mathbf{k b} \mathbf{- 1} \mathbf{M b}$ & $>\mathbf{1 ~ M b}$ \\
\hline Benign and CNVs of unknown clinical significance & 239 & 179 & 52 & 8 \\
\hline Benign CNVs & 118 & 90 & 21 & 7 \\
\hline CNVs of unknown clinical significance & 121 & 89 & 31 & 1 \\
\hline
\end{tabular}

CNVs: copy number variations.

size as follows: $90(76.3 \%)$ covered $100-500 \mathrm{~Kb} ; 21(17.8 \%)$ were from $500 \mathrm{~Kb}$ to $1 \mathrm{Mb}$; seven (5.9\%) were larger than $1 \mathrm{Mb}$. There were 27 duplications and 91 deletions (Table $2)$. The $\mathrm{CNV}$ s of unknown clinical significance numbered 121 and were of the following size: 89 (73.5\%) from 100 to $500 \mathrm{~Kb} ; 31(25.6 \%)$ were from $500 \mathrm{~Kb}$ to $1 \mathrm{Mb}$; one $(0.8 \%)$ was a size larger than $1 \mathrm{Mb}$. Copy number variations (excluding the pathogenic ones) were present in 74 patients. In 70 persons, the size of CNVs ranged from 100 to $500 \mathrm{~Kb}$; in $39 \mathrm{CNVs}$ were from $500 \mathrm{~Kb}$ to $1 \mathrm{Mb}$, and in seven larger than $1 \mathrm{Mb}$. In the majority of the cases, a combination of benign CNVs and CNVs of unknown clinical significance was defined. It is noteworthy that in our sample, CNVs ranging in size between 100 and 500 $\mathrm{kb}$ were of greater frequency.

\section{DISCUSSION}

In 31 patients, 41 pathogenic variations were found. In six of these patients, eight pathogenic aberrations associated with the corresponding phenotype were defined. The interpretation of other variations was made on the basis of the frequency in the studied group, the size of the variations, content of genes in the region and the type of the CNVs (deletion or duplication).

The CNVs with a $1.0-3.0 \%$ frequency in the population are accepted as benign polymorphisms. This gave us reason to consider as probably benign, those varia- tions that occurred at frequencies higher than $1.2 \%$ in the studied group. Twenty-four of the CNVs conform with these criteria and were therefore considered to probably be benign. Abnormal variations with a frequency higher than $1.2 \%$, were found in the following chromosomal loci: $2 q 13(2.5 \%), 3 q 29(2.5 \%), 7 q 11.23$ (3.7\%), 8p23.1 (8.6\%) and Xp22.33 (18.5\%).

The deletion in the $(2)(\mathrm{p} 16.3)$ region, found in patient 79, was pathogenic and covered one $H G N C$ gene $(N R X N 1)$ and one OMIM gene (NRXN1). It covered the OMIM loci associated with autism. The detected deletion in the (5) (q35.2q35.3) region in the same patient was also pathogenic and covered $40 H G N C$ and 24 OMIM genes among which was the NSD1, whose loss of function was considered a major cause of Sotos syndrome. The NSD1 protein controls the activity of genes related to normal growth and development, although many of these genes have not been identified [33]. The described pathogenic CNVs in this gene were more than 100 . Sotos syndrome is characterized by facial dysmorphism, dolichocephaly, cognitive decline, mild-to-severe intellectual disability and overgrowth. In the second patient (\#52), aCGH revealed a pathogenic deletion, del(10)(q26.12q26.3), covering 85 $H G N C$ and 49 OMIM genes. This type of chromosomal aberration leads to phenotypic manifestations such as facial dysmorphism, postnatal growth retardation, developmental delay, intellectual disability, hypotonia in newborns, feeding disorders, microcephaly, digital anomalies, heart 
defects and defects of the genitourinary system. The common feature of the patients with these aberrations is that they have behavioral problems. Some sources considered four candidate genes: CTBP2, ADRB1, DPYSL4, DRD1IP, associated with neural development and function [34].

The pathogenic deletion in patient 46, del(4) (q34.3q35.2), included 49 HGNC and 21 OMIM genes and the duplication, also pathogenic, on chromosome 12, dup(12) (p13.33p13.1), covered $226 H G N C$ and 156 OMIM genes. The presence of terminal aberrations is very often a result of family rearrangements, but due to lack of samples from the parents, the origin of the detected aberrations was not set. The clinical features included facial dysmorphism, obesity, increased appetite and other symptoms.

Patient 41 was revealed to carry pathogenic deletion 17 , $\operatorname{del}(17)(q 12 q 12)$, covering $24 H G N C$ and 24 OMIM genes, which are associated with renal cysts, diabetic syndrome, developmental delay, autism and schizophrenia, seizures and less common phenotypes with dysmorphic features, transitory neonatal hypercalcemia, Müller aplasia and congenital diaphragmatic hernia. An important candidate gene was $H N F 1 B$ associated with the development of the kidneys and pancreas.

The detected deletion on chromosome 22, del(22) (q11.21q11.22), in patient 35, was also pathogenic covering $68 \mathrm{HGNC}$ and 12 OMIM genes and affecting many organs and systems. It was assumed that the majority of the symptoms (heart defects, cleft palate, facial dysmorphism, hearing loss, hypocalcaemia), were caused by deletion of the $T B X 1$ gene.

The last detected pathogenic aberration was a duplication in chromosome 15, $\operatorname{dup}(15)(\mathrm{q} 11.2 \mathrm{q} 13.1)$, which included 111 HGNC and 16 OMIM genes. This syndrome is represented by autism, intellectual disability, muscular hypotonia, ataxia, seizures, developmental delay and behavioral problems [35]. According to Tan et al. [36], at least three genes (NIPA1, NIPA2, CYFIP1), located in this region were associated with the development of $\mathrm{CNS}$ (central nervous system). A duplication of the copy of the $S N R P N$ gene obtained from the mother is associated with autism. Other important genes associated with the development and neurological disorders are GABRA5, GABRA3, GABRG3, MAGEL2, MKRN3, NDN, SNRPN and UBE3A. The first three encode subunits of the GABA-receptors that mediate the main inhibitory neurotransmitter in the brain (GABA) [36].

The total number of established benign CNVs and CNVs of unknown clinical significance, amounted to 239, 141 of which were deletions and 98 were duplications. The total number of variations (with pathogenic ones) was
280 , an average of 3.5 variations per patient. Two large studies in 2004 revealed that the genome of each person contains approximately 12 CNVs [27,28]. Later, in 2009, Itsara et al. [19] analyzed 2500 controls and found that the average number of CNVs for one person is between three and seven variations. Our data is very similar to that of Itsara et al. [19].

In more than one patient, benign CNVs were identified in the following cytogenetic loci: 8p11.22, 6p25.3, 1q21.2, $2 \mathrm{q} 37.3,10 \mathrm{q} 11.22,14 \mathrm{q} 11.2,5 \mathrm{q} 13.2,10 \mathrm{q} 26.3,15 \mathrm{q} 11.2$, $19 \mathrm{q} 13.31$, and two different $\mathrm{CNV}$ s were detected in region 8p23.1. In six patients, six single variations in the loci were found: $14 \mathrm{q} 32.33,10 \mathrm{q} 11.22,5 \mathrm{p} 15.33,16 \mathrm{p} 13.11,15 \mathrm{q} 14$, 14q21.2 (Figure 1).

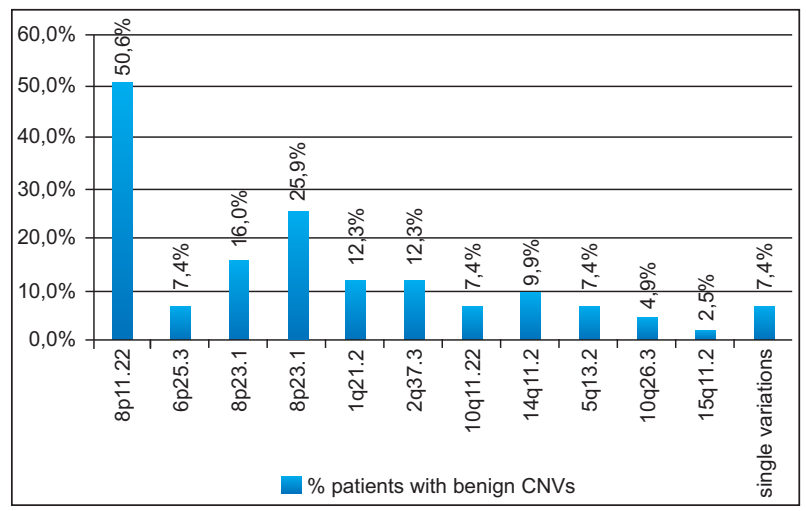

Figure 1. Frequency of various benign $\mathrm{CNVs}$ in the studied group.

The most common benign CNVs were found in over $10.0 \%$ of the tested patients and were at the following locations: 8 p11.22 and 1q21.2, and two different variations in the 8p23.1 locus. Deletions were identified in some of the cases, while in others, duplications, which indicated that these areas contained a significant number of variations. To establish the actual frequency of the detected unique benign CNVs, it was better to perform a genomic screening of a larger group of patients. According to Kooy [37], polymorphic $\mathrm{CNV}$ s are part of the natural genetic differences in humans, while rare variants can be related to certain disorders. A large study found that polymorphic CNVs are probably not closely related to the genetic etiology of multifactorial diseases, and it is possible that they are part of the benign genetic variations between individuals [38].

The number of variants of unknown clinical significance was 121 . They were distributed by size as follows: 89 (73.6\%) 100-500 kb; 31 (25.6\%) $500 \mathrm{~kb}-1 \mathrm{Mb}$; one $(0.8 \%)$ larger than $1 \mathrm{Mb}$. There were 50 deletions and 71 duplications. From the identified 121 variations of unknown clinical significance, in the following cytogenetic loci: 8p11.22, 8p23.1, 14q32.33, Xp22.33, 17q21.31, 16p11.2, 
$2 q 37.3,14 q 11.2,22 q 11.23,11 q 25,12 p 13.31$, Xq22.3, $2 \mathrm{p} 11.1$, CNVs were found in more than one patient. There were 33 single CNVs detected in 27 patients.

Our results revealed a high rate of CNVs of unknown clinical significance. It is very likely that some of these variations are benign for the Bulgarian population. It is well known that the Bulgarian nation has a great genetic heterogeneity. Thirteen types of CNVs of unknown clinical significance were defined. They were located as follows: 8p11.22, 8p23.1, 14q32.33, Xp22.33, 17q21.31, 16p11.2, 2q37.3, 14q11.2, 22q11.23, 11q25, 12p13.31, Xq22.3, 2p11.1 ( 88 of the total number), and occurred in over $1.2 \%$ of the patients. That gave us reason to assume that they had no pathogenic nature. The most common, probably benign CNVs in our study, were in loci 14q32.33, 17q21.31 and $16 \mathrm{p} 11.12$, occurring in over $15.0 \%$ of the patients. Thirteen of the CNVs detected in loci 14q32.33, 16p11.2, 16p12.3, 17q21.31, 1q21.2, 20p12.1, 22q11.22, 2q37.3, 4q35.2, 8 p23.1 were found in patients with already defined pathogenic aberrations of major size, and associated with the corresponding phenotype. According to some sources, in the presence of large chromosomal aberrations associated with a particular phenotype, it is unlikely for the identified smaller rearrangement to be essential for the development of the disease [23]. Therefore we assumed that it was possible for these 13 variants to have no relation with the clinical phenotype. In patient 30 a duplication of unknown clinical significance was identified in the 1q21.2 region, which contained $15 H G N C$ and 1 OMIM genes. Among them was the NBPF23 gene, which belongs to a gene family, characterized by tandem repeats of the DUF1220 protein domains. Several developmental problems and neurogenetic disorders such as microcephaly, macrocephaly, autism, schizophrenia, learning disorders, congenital heart disease, neuroblastoma, congenital anomalies of the kidney and urinary tract [39], are associated with identified CNVs located in the region with more DUF1220 domains. Based on this information, we could not exclude the potential pathogenic effect of the CNVs in this region. Nineteen of the rest of the CNVs of unknown clinical significance could not be interpreted as probably benign. We need more complementary studies to clarify the phenotypic effect.

Five types of the pathogenic CNVs, located in chromosomal loci 2q13, 3q29, 8p23.1, 7q11.23, Xp22.33 (24 variations) were found in a high percentage (over 1.2\%) of the patients. Their high frequencies gave us reason to suppose that despite their pathogenic nature, these variations might be considered to be CNVs of unknown clinical significance or probably benign. In four patients, we identified four pathogenic variations with no clinical significance, in the following chromosomal loci: 22q11.22, 8p23.2, 2q13, 4q12. To determine participation in the variation genes, we made a reference to the Database of Genomic Variants (DGV). The $O M I M$ genes responsible for the clinical phenotype were found in none of the four CNVs.

In the selected group of patients with congenital anomalies, $280 \mathrm{CNVs}$ have been proven, respectively 41 pathogenic, 118 benign and 121 of unknown clinical significance. We found $13.6 \%$ incidence of pathogenic CNVs by aCGH. Eight pathogenic variations corresponding to the clinical phenotype of the patients, were identified. We revealed genotype-phenotype correlations between many genes and the manifested clinical features. During the interpretation of the CNVs of unknown clinical significance and the pathogenic ones, an additional category was defined, "probably benign variations." It was concluded that there is a need to apply new genomic technologies with greater resolution, as a significant proportion of the patients were left with an unclear diagnosis. This would facilitate the detection and interpretation of the genomic aberrations in order to make an accurate diagnosis and to optimize therapeutic approaches.

\section{ACKNOWLEDGMENTS}

We thank Dr. Theodor Zamfirov (Manager) and Elta 90 M Ltd., Sofia, Bulgaria, for their financial support relating to the publication of this study.

Declaration of Interest. The authors report no conflicts of interest. The authors alone are responsible for the content and writing of this article.

\section{REFERENCES}

1. Pennisi E. Breakthrough of the year. Human genetic variation. Science. 2007; 318(5858): 1842-1843.

2. Aldred PM, Hollox EJ, Armour JA. Copy number polymorphism and expression level variation of the human $\alpha$-defensin genes DEFA1 and DEFA3. Hum Mol Genet. 2005; 14(14): 2045-2052.

3. Gonzalez E, Kulkarni H, Bolivar H, Mangano A, Sanchez R, Catano G, et al. The influence of CCL3L1 gene-containing segmental duplications on HIV-1/ AIDS susceptibility. Science. 2005; 307(5714): 14341440.

4. Hollox EJ, Armour JA, Barber JC. Extensive normal copy number variation of a $\beta$-defensin antimicrobialgene cluster. Am J Hum Genet. 2003; 73(3): 591-600. 
5. Linzmeier RM, Ganz T. Human defensin gene copy number polymorphisms: Comprehensive analysis of independent variation in $\alpha$ - and $\beta$-defensin regions at 8p22-p23. Genomics. 2005; 86(4): 423-430.

6. McCarroll SA, Hadnott TN, Perry GH, Sabeti PC, Zody MC, Barrett JC, et al. Common deletion polymorphisms in the human genome. Nat Genet. 2006; 38(1): 86-92.

7. Perry GH, Dominy NJ, Claw KG, Lee AS, Fiegler $\mathrm{H}$, Redon R, et al. Diet and the evolution of human amylase gene copy number variation. Nat Genet. 2007; 39(10): 1256-1260.

8. Stranger BE, Forrest MS, Dunning M, Ingle CE, Beazley $\mathrm{C}$, Thorne N, et al. Relative impact of nucleotide and copy number variation on gene expression phenotypes. Science. 2007; 315(5813): 848-853.

9. Aitman TJ, Dong R, Vyse TJ, Norsworthy PJ, Johnson $\mathrm{MD}$, Smith J, et al. Copy number polymorphism in Fcgr3 predisposes to glomerulonephritis in rats and humans. Nature. 2006; 439(7078): 851-855.

10. Fanciulli M, Norsworthy PJ, Petretto E, Dong R, Harper L, Kamesh L, et al. FCGR3B copy number variation is associated with susceptibility to systemic, but not organ-specific, autoimmunity. Nat Genet. 2007; 39(6): 721-723.

11. Fellermann K, Stange DE, Schaeffeler E, Schmalzl H, Wehkamp J, Bevins CL, et al. A chromosome 8 gene-cluster polymorphism with low human $\beta$-defensin 2 gene copy number predisposes to Crohn disease of the colon. Am J Hum Genet. 2006; 79(3): 439-448.

12. Gamazon ER, Nicolae DL, Cox NJ. A study of CNVs as trait-associated polymorphisms and as expression quantitative trait loci. PLoS Genet. 2011; 7(2): e1001292.

13. Hollox EJ, Huffmeier U, Zeeuwen PL, Palla R, Lascorz J, Rodijk-Olthuis D, et al. Psoriasis is associated with increased $\beta$-defensin genomic copy number. Nat Genet. 2008; 40(1): 23-25.

14. Park J, Chen L, Ratnashinge L, Sellers TA, Tanner JP, Lee JH, et al. Deletion polymorphism of UDPglucuronosyltransferase $2 \mathrm{~B} 17$ and risk of prostate cancer in African American and Caucasian men. Cancer Epidemiol Biomarkers Prev. 2006; 15(8): 1473-1478.

15. Yang Y, Chung EK, Wu YL, Savelli SL, Nagaraja $\mathrm{HN}$, Zhou B, et al. Gene copy-number variation and associated polymorphisms of complement component C4 in human systemic lupus erythematosus (SLE): Low copy number is a risk factor for and high copy number is a protective factor against SLE susceptibil- ity in European Americans. Am J Hum Genet. 2007; 80(6): 1037-1054.

16. Abyzov A, Urban AE, Snyder M, Gerstein M. CNVnator: An approach to discover, genotype, and characterize typical and atypical CNVs from family and population genome sequencing. Genome Res. 2011; 21(6): 974-984.

17. Campbell CD, Sampas N, Tsalenko A, Sudmant PH, Kidd JM, Malig M, et al. Population-genetic properties of differentiated human copy-number polymorphisms. Am J Hum Genet. 2011; 88(3): 317-332.

18. Fu Y, Chen Z, Blakemore AI, Orwoll E, Cohen DM. Absence of AVPR2 copy number variation in eunatremic and dysnatremic subjects in non-Hispanic Caucasian populations. Physiol Genomics. 2010; 40(3): 121-127.

19. Itsara A, Cooper GM, Baker C, Girirajan S, Li J, Absher $\mathrm{D}$, et al. Population analysis of large copy number variants and hotspots of human genetic disease. Am J Hum Genet. 2009; 84(2): 148-161.

20. Pinto D, Marshall C, Feuk L, Scherer SW. Copynumber variation in control population cohorts. Hum Mol Genet. 2007; 16(Spec No. 2): R168-R173.

21. Zhang LN, Meng Z, He ZW, Li DF, Luo XY, Liang LY. [Clinical phenotypes and copy number variations in children with microdeletion and microduplication syndromes: An analysis of 50 cases]. Zhongguo Dang Dai Er Ke Za Zhi. 2016; 18(9): 840-845.

22. Feuk L, Carson AR, Scherer SW. Structural variation in the human genome. Nat Rev Genet. 2006; 7(2): 85-97.

23. Lee C, Iafrate AJ, Brothman AR. Copy number variations and clinical cytogenetic diagnosis of constitutional disorders. Nat Genet. 2007; 39(7 Suppl): S48-S54.

24. Freeman JL, Perry GH, Feuk L, Redon R, McCar-roll SA, Altshuler DM, et al. Copy number variation: New insights in genome diversity. Genome Res. 2006; 16(8): 949-961.

25. Scherer SW, Lee C, Birney E, Altshuler DM, Eichler EE, Carter NP, et al. Challenges and standards in integrating surveys of structural variation. Nat Genet. 2007; 39(7 Suppl): S7-S15.

26. Alaerts M, Del-Favero J. Searching genetic risk factors for schizophrenia and bipolar disorder: Learn from the past and back to the future. Hum Mutat. 2009; 30(8): 1139-1152.

27. Iafrate AJ, Feuk L, Rivera MN, Listewnik ML, Donahoe PK, Qi Y, et al. Detection of large-scale variation in the human genome. Nat Genet. 2004; 36(9): 949-951. 
28. Sebat J, Lakshmi B, Troge J, Alexander J, Young J, Lundin P, et al. Large-scale copy number polymorphism in the human genome. Science. 2004; 305(5683): 525-528.

29. Redon R, Ishikawa S, Fitch KR, Feuk L, Perry GH, Andrews TD, et al. Global variation in copy number in the human genome. Nature. 2006; 444(7118): $444-$ 454.

30. Levy S, Sutton G, Ng PC, Feuk L, Halpern AL, Walenz $\mathrm{BP}$, et al. The diploid genome sequence of an individual human. PLoS Biol. 2007; 5(10): e254.

31. Venter JC. Multiple personal genomes await. Nature. 2010; 464(7289): 676-677.

32. Caramaschi E, Stanghellini I, Magini P, Giuffrida MG, Scullin S, Giuva T, et al. Predictive diagnostic value for the clinical features accompanying intellectual disability in children with pathogenic copy number variations: A multivariate analysis. Ital J Pediatr. 2014; 40: 39. doi: 10. 1186/1824-7288-40-39.

33. Choufani S, Cytrynbaum C, Chung BH, Turinsky AL, Grafodatskaya D, Chen YA, et al. NSD1 mutations generate a genome-wide DNA methylation signature. Nat Commun. 2015; 6: 10207. doi: 10.1038/ ncomms 10207.
34. Courtens W, Wuyts W, Rooms L, Pera SB, Wauters J. A subterminal deletion of the long arm of chromosome 10: A clinical report and review. Am J Med Genet A. 2006; 140(4): 402-409.

35. Kalsner L, Chamberlain SJ. Prader-Willi, Angelman, and 15q11-q13 duplication syndromes. Pediatr Clin North Am. 2015; 62(3): 587-606.

36. Tan ES, Yong MH, Lim EC, Li ZH, Brett MS, Tan EC. Chromosome 15q11-q13 copy number gain detected by array-CGH in two cases with a maternal methylation pattern. Mol Cytogenet. 2014; 7: 32. doi: 10.1186/17558166-7-32.

37. Kooy RF. Distinct disorders affecting the brain share common genetic origins. F1000 Biol Rep. 2010; 2: 11. doi: 10.3410/B2-11. (http://f1000.com/reports/b/2/11.)

38. Craddock N, Hurles ME, Cardin N, Pearson RD, Plagnol $\mathrm{V}$, Robson $\mathrm{S}$, et al. Genome-wide association study of CNVs in 16,000 cases of eight common diseases and 3,000 shared controls. Nature. 2010; 464(7289): 713-720.

39. Diskin SJ, Hou C, Glessner JT, Attiyeh EF, Laudenslager $\mathrm{M}$, Bosse $\mathrm{K}$, et al. Copy number variation at 1q21.1 associated with neuroblastoma. Nature. 2009; 459(7249): 987-991. 
